\title{
Progress and prospects of lattice supersymmetry
}

\section{David Schaich ${ }^{* \dagger}$}

AEC Institute for Theoretical Physics, University of Bern, 3012 Bern, Switzerland

E-mail: schaicheitp.unibe.ch

\begin{abstract}
Supersymmetry plays prominent roles in the study of quantum field theory and in many proposals for potential new physics beyond the standard model, while lattice field theory provides a nonperturbative regularization suitable for strongly interacting systems. Lattice investigations of supersymmetric field theories are currently making significant progress, though many challenges remain to be overcome. In this brief overview I discuss particularly notable progress in three areas: supersymmetric Yang-Mills (SYM) theories in fewer than four dimensions, as well as both minimal $\mathcal{N}=1 \mathrm{SYM}$ and maximal $\mathcal{N}=4 \mathrm{SYM}$ in four dimensions. I also highlight super-QCD and sign problems as prominent challenges that will be important to address in future work.
\end{abstract}

The 36th Annual International Symposium on Lattice Field Theory (LATTICE2018)

22-28 July 2018

Michigan State University, East Lansing, Michigan, United States

\footnotetext{
${ }^{*}$ Speaker.

${ }^{\dagger}$ Participation supported by a travel grant from the Faculty of Science at the University of Bern.
} 


\section{Introduction, motivation and background}

Supersymmetry (susy) plays prominent roles in modern theoretical physics, as a tool to improve our understanding of quantum field theory (QFT), as an ingredient in new physics models, and as a means to study quantum gravity via holographic duality. Lattice field theory provides a non-perturbative regularization for QFTs, and other contributions to these proceedings document the prodigious success of this framework applied to QCD and similar theories. It is natural to consider using lattice field theory to investigate supersymmetric QFTs, especially in strongly coupled regimes. Here I review the recent progress and future prospects of lattice studies of supersymmetric systems, focusing on $4 \mathrm{~d}$ gauge theories and their dimensional reductions to $d<4$.

Lattice susy now has more than four decades of history [1-7]. Unfortunately, progress in this field has been slower than for QCD, in large part because the lattice discretization of space-time breaks susy. This occurs in three main ways. First, the anti-commutation relation

$\left\{Q_{\alpha}, \bar{Q}_{\dot{\alpha}}\right\}=2 \sigma_{\alpha \dot{\alpha}}^{\mu} P_{\mu}$ in the super-Poincaré algebra connects the spinorial generators of susy transformations to the generator of infinitesimal translations, which do not exist in discrete space-time. Next, bosonic and fermionic fields are typically discretized differently on the lattice, preventing susy transformations from correctly interchanging superpartners. Finally, susy requires a derivative operator that obeys the Leibniz rule [1], which is violated by standard lattice finite-difference operators. 'No-go theorems' [8,9] establish that only non-local derivative and product operators can obey the Leibniz rule in discrete space-time. Efforts continue to construct alternate formulations that may better balance locality and susy [10 - 13]. These formulations are complicated and so far limited to systems without gauge invariance, either in $(0+1)$ dimensions or on infinite lattices.

Broken susy means quantum effects in the lattice calculation will generate susy-violating operators. These include relevant operators for which counterterms will have to be fine-tuned in order to recover the supersymmetric QFT of interest in the $a \rightarrow 0$ continuum limit that removes the UV cutoff $a^{-1}$. In theories with scalar fields (squarks or scalars in the $\mathcal{N}>1$ gauge supermultiplet with extended susy), these scalars' mass terms present fine-tuning problems similar to that of the Higgs boson in the standard model. Additional susy-violating operators include fermion (quark and gaugino) mass terms, Yukawa couplings, and quartic (four-scalar) terms. Altogether there are typically $\mathcal{O}(10)$ of these operators $[3,14,15]$, implying such high-dimensional parameter spaces that there seems to be little hope of effectively navigating them in numerical lattice calculations.

The following three sections focus on three different ways to reduce the amount of fine-tuning in lattice studies of supersymmetric Yang-Mills (SYM) theories. We begin in the next section by reviewing dimensional reductions to SYM theories in fewer than four space-time dimensions, which has received the most attention from the community so far. We return to four dimensions in Sec. 2, considering first the special case of minimal $(\mathcal{N}=1)$ SYM, which is vastly simplified by the absence of scalar fields. Another special case in four dimensions is maximal $(\mathcal{N}=4) \mathrm{SYM}$, the topic of Sec. 3, for which a closed subalgebra of the supersymmetries can be preserved at nonzero lattice spacing, again drastically reducing the necessary fine-tuning. Finally, Sec. 4 briefly discusses some prominent challenges to be faced in the future.

\section{Lower-dimensional systems}

Dimensionally reduced SYM theories can be much easier to analyze numerically. In addition 
to the smaller number of degrees of freedom for an $L^{d}$ lattice, the resulting lower-dimensional theories tend to be super-renormalizable and in many cases a one-loop counterterm calculation suffices to restore susy in the continuum limit [16-18]. We will label systems by their number of supercharges: $Q=4,8$ or 16 corresponding to $\mathcal{N}=1,2$ or $4 \mathrm{SYM}$ in four dimensions (or to minimal SYM in $d=4,6$ or 10 dimensions). For $d \leq 4$ these theories involve a gauge field, $Q$ fermionic component fields, and $4-d, 6-d$ or $10-d$ real scalar fields, respectively, all of which are massless and transform in the adjoint rep of gauge group $\mathrm{SU}(N)$ or $\mathrm{U}(N)=\mathrm{SU}(N) \otimes \mathrm{U}(1)$.

0+1 dimensions: The reduction to 'SYM quantum mechanics' $(\mathrm{QM})$ has been the subject of many numerical studies over the past decade, starting with Refs. [19, 20]. These systems involve balanced collections of interacting bosonic and fermionic $N \times N$ matrices at a single spatial point. One reflection of the simplicity of SYM QM is that a lattice regularization may not even be required; a gauge-fixed Monte Carlo approach employing a hard momentum cutoff [19] was used by Refs. [21-26]. Another illustration is a recent proposal [27] that 'ungauging' $Q=16 \mathrm{SYM}$ QM (to consider a scalar-fermion system with $\mathrm{SU}(N)$ global symmetry) has relatively little effect, in the sense that both the gauged and ungauged models flow to the same theory in the IR. This conjecture was quickly tested by lattice calculations that found consistent results [28].

Even though these quantum-mechanical systems are much simpler to study on the lattice than their 4d SYM counterparts, they remain computationally non-trivial. This is demonstrated by the state-of-the-art results for $Q=16$ SYM QM shown in Fig. 8 of Ref. [29]. This $Q=16$ case has attracted particular interest due to its connections to string theory [30], and especially the conjecture [31] that the large- $N$ limit of this system describes the strong-coupling ('M-theory') limit of type-IIA string theory in light-front coordinates [32,33]. At finite temperature, this conjecture relates the large- $N$ limit of the (deconfined) $Q=16$ system to a dual compactified $11 \mathrm{~d}$ black hole geometry in M-theory, and Fig. 8 of Ref. [29] shows the dual black hole internal energy determined from lattice SYM QM computations. This was previously studied numerically by Refs. [21, 23, 26, 34-38].

Ref. [29] improves upon the earlier work by carrying out controlled extrapolations to the large- $N$ continuum limit, allowing for more robust comparisons with dual gravitational predictions. Fig. 7 of Ref. [29] shows one such extrapolation, for a fixed value $T=0.5$ of the dimensionless temperature $T \equiv t_{\operatorname{dim}} / \lambda_{\operatorname{dim}}^{1 / 3}$. (The 't Hooft coupling $\lambda_{\operatorname{dim}}=g^{2} N$ has dimension $\left[\lambda_{\text {dim }}\right]=4-d$.) With fixed $T$ the continuum limit corresponds to extrapolating the number of lattice sites $L \rightarrow \infty$. At low temperatures the results in Fig. 8 of Ref. [29] convincingly approach the leading-order gravitational prediction from classical supergravity (SUGRA), providing nonperturbative first-principles evidence that the holographic duality conjecture is correct. In addition, the growing difference between the lattice results and the SUGRA curve at higher temperatures can be considered a prediction of higher-order quantum gravitational effects that are enormously difficult to calculate analytically.

The computational non-triviality of these investigations comes primarily from the large values of $N$ that are needed ( $16 \leq N \leq 32$ in Ref. [29], large enough to benefit from dividing individual $N \times N$ matrices across multiple MPI processes via the MMMM code). The computational cost of $N \times N$ matrix multiplication scales $\propto N^{3}$, compared to the $\sim L^{5 d / 4}$ costs of the rational hybrid Monte Carlo (RHMC) algorithm. In addition to improving control over the $N \rightarrow \infty$ extrapolations, 
large values of $N \gtrsim 10$ are also required to suppress a thermal instability associated with the noncompact quantum moduli space of $Q=16 \mathrm{SYM}$ QM [35]. For sufficiently low temperatures and sufficiently small $N$ the system is able to run away along these flat directions (holographically interpreted as D0-brane radiation from the dual black hole). Formally a scalar potential should be added to the lattice action to stabilize the desired vacuum, and then removed in the course of the continuum extrapolation, further increasing computational costs [35, 39]. However, Ref. [29] argues that in practice it is possible to carry out Monte Carlo sampling around a metastable vacuum so long as $N$ is sufficiently large. In particular, $N$ must increase in order to reach smaller $T$.

Further numerical investigations of SYM QM systems are underway [40-44]. At the same time, the good control over the necessary extrapolations that has now been achieved for the $Q=$ 16 case also motivates pursuing comparable quality in lattice studies of less-simplified systems. One example of such a system is the Berenstein-Maldacena-Nastase (BMN) deformation of $Q=$ 16 SYM QM [45], which introduces a non-zero mass for the 9 scalars and 16 fermions while preserving all 16 supercharges. This has been studied numerically by Refs. [46-48]. The mass deformation explicitly breaks the $\mathrm{SO}(9)$ global symmetry to $\mathrm{SO}(6) \times \mathrm{SO}(3)$. It also lifts the flat directions, serving as a supersymmetric regulator that need not be removed in the continuum limit.

In addition, the mass parameter $\mu$ provides a second axis for the finite-temperature phase diagram, as shown in Fig. 5 of Ref. [48]. As $\mu \rightarrow \infty$ the theory becomes gaussian, and the deconfinement temperature $T_{d}$ can be computed perturbatively in $1 / \mu$. At small $\mu$, Ref. [49] carried out the numerical construction of the SUGRA black hole geometry dual to the deconfined phase, predicting $T_{d}$ to linear order in $\mu$. Figure 5 of Ref. [48] shows recent numerical results in reasonably good agreement with these predictions, given the fixed $N=8$ and $L=24$. In addition to the deconfinement transition signalled by the Polyakov loop, this work observes a transition between an approximately $\mathrm{SO}(9)$-symmetric phase at high temperatures and an $\mathrm{SO}(6) \times \mathrm{SO}(3)$ phase at low temperatures. For small $\mu \lesssim 3$ these transitions occur at the same $T_{d}$, while at larger $\mu$ higher temperatures are needed to recover approximate $\mathrm{SO}(9)$ symmetry. It will be interesting to systematize large- $N$ continuum extrapolations in future lattice BMN investigations, since these turned out to be significant in the $\mu=0$ limit discussed above.

1+1 dimensions: Dimensional reductions of SYM to $d=2$ and 3 also provide less-simplified systems compared to SYM QM, while still being significantly more tractable than $d=4$. Although there has been a lot of work in this area over the years, much of the effort has focused on constructing clever lattice formulations that minimize fine-tuning in principle, rather than using these constructions in practical numerical calculations. Here we will highlight the numerical calculations, which leaves little to say about $d=3$ : see Refs. [18, 50, 51] for $Q=8$ formulations.

The main clever constructions that have been applied are based on either 'twisting' [52 -54] or orbifolding [55-58], two approaches that actually produce equivalent constructions [59, 60]. (See Ref. [2] for a thorough review.) Here we discuss only the twisting approach, which identifies at most $\left\lfloor Q / 2^{d}\right\rfloor$ linear combinations of supercharges, $\mathcal{Q}$, that are nilpotent, $\mathcal{Q}^{2}=0$. These are found by organizing the $Q$ supercharges into irreps of a 'twisted rotation group' $\mathrm{SO}(d)_{\mathrm{tw}} \equiv$ $\operatorname{diag}\left[\mathrm{SO}(d)_{\text {euc }} \otimes \mathrm{SO}(d)_{R}\right]$, where $\mathrm{SO}(d)_{\text {euc }}$ is the Wick-rotated Lorentz group and $\mathrm{SO}(d)_{R}$ is a global $R$-symmetry. The nilpotent $\mathcal{Q}$ are those that transform in the twisted-scalar rep. The requirement $Q \geq 2^{d}$ ensures a sufficiently large $R$-symmetry. This procedure provides a closed susy 
subalgebra $\{\mathcal{Q}, \mathcal{Q}\}=0$ at non-zero lattice spacing, leading to a $\mathcal{Q}$-invariant lattice action with no need of the Leibniz rule.

For some theories there are multiple ways the twisting procedure can be carried out. One approach [59, 61 -63] combines the gauge and scalar fields into a complexified gauge field, leading to $\mathrm{U}(N)=\mathrm{SU}(N) \otimes \mathrm{U}(1)$ gauge invariance and non-compact lattice gauge links $\{\mathcal{U}, \overline{\mathcal{U}}\}$ with a flat measure. The fermion fields are twisted in the same way as the supercharges, obtaining the same lattice gauge transformations as the bosonic degrees of freedom. Although the U(1) sector decouples in the continuum, at non-zero lattice spacing it can introduce unwanted artifacts at strong coupling, and ongoing work is searching for good ways to suppress these [63-65]. For $d=2$ a different approach $[52,53,66-70]$ works with compact gauge links and gauge group $\mathrm{SU}(N)$, at the cost of imposing an admissibility condition to resolve a huge degeneracy of vacua (but see Ref. [71]), which becomes more problematic in higher dimensions [72, 2]. This formulation has been used by several numerical studies of the $Q=4[39,73-80]$ and $Q=16[81,82]$ theories.

Fig. 8 of Ref. [64] shows recent results from Ref. [64] for the phase diagram of $2 \mathrm{~d} Q=$ 16 SYM, using the non-compact twisted construction described above [83-86]. The system is formulated on an $r_{L} \times r_{\beta}$ torus, with $r_{\beta}=1 / T$ the inverse dimensionless temperature while $r_{L}=$ $L_{\operatorname{dim}} \sqrt{\lambda_{\operatorname{dim}}}$ is the corresponding dimensionless length of the spatial cycle. At high temperatures (small $r_{\beta}$ ), the fermions pick up a large thermal mass and the system reduces to a $1 \mathrm{~d}$ bosonic QM. In this limit (at large $N$ ), Refs. [87-90] predict a 'spatial deconfinement' transition as $r_{L}$ decreases, signalled by a non-zero spatial Wilson line $\operatorname{Tr}\left[\prod_{x_{i}} \mathcal{U}_{x}\left(x_{i}, t\right)\right]$.

In the low-temperature (large- $r_{\beta}$ ) limit, there is a large- $N$ holographic prediction for a similar transition. Here the large- $r_{L}$ spatially confined phase is conjectured to be dual to a homogeneous black string with a horizon wrapping around the spatial cycle, while the small- $r_{L}$ spatially deconfined phase corresponds to a localized black hole. As in the BMN case, the holographic analyses require challenging numerical SUGRA constructions [91] of these dual black hole and black string geometries. The lattice results for the spatial deconfinement transition (with $N=12$ and fixed lattice sizes $32 \times 4,24 \times 4,24 \times 6,24 \times 9,24 \times 12$ and $18 \times 12$ ) reproduce the high-temperature bosonic QM expectations quite well and are consistent with holography at lower temperatures, albeit with rapidly increasing uncertainties. At low temperatures a scalar potential is added to the lattice action and then extrapolated to zero in order to avoid the thermal instability mentioned above for SYM QM. Ref. [64] also calculates the internal energies of the dual black hole and black string, in both phases finding consistency with holographic expectations within large uncertainties. It will be interesting to see future work improve upon these results, ideally accessing lower temperatures in addition to gaining control over extrapolations to the large- $N$ continuum limit.

2d SYM also possesses rich zero-temperature dynamics that are important to explore nonperturbatively, in addition to studying the thermal behavior discussed above. Refs. [92, 93] argue that the 'meson' spectrum of the $Q=4$ theory should include a massless supermultiplet, unlike the $4 \mathrm{~d} \mathcal{N}=1 \mathrm{SYM}$ of which this is the dimensional reduction. A recent lattice calculation using straightforward Wilson fermions observes such a massless multiplet [94], and also checks for spontaneous susy breaking (SSB), which Ref. [95] suggests might occur for this theory. No evidence of SSB is seen, consistent with Ref. [96] and older work [74, 75, 78] using twisted formulations. 


\section{Minimally supersymmetric Yang-Mills $(\mathcal{N}=1 \mathbf{S Y M})$ in four dimensions}

Returning to four dimensions, we can note that most of the susy-violating operators involve scalar fields, viz. the scalar mass terms, Yukawa couplings, and quartic operators. This implies a vast reduction of fine-tuning for $\mathcal{N}=1 \mathrm{SYM}$, the only $4 \mathrm{~d}$ supersymmetric gauge theory with no scalar fields. This theory consists of a $\mathrm{SU}(N)$ gauge field and its superpartner gaugino, a massless Majorana fermion transforming in the adjoint rep of $\mathrm{SU}(N)$. The only relevant (or marginal) operator that may need to be fine-tuned to obtain the correct continuum limit is the gaugino mass [97, 98]. We can even avoid this single fine-tuning by working with Ginsparg-Wilson (GW; overlap or domain-wall) lattice fermions that preserve chiral symmetry and protect the gaugino mass against large additive renormalization. Although the axial anomaly breaks the classical $\mathrm{U}(1) R$-symmetry of $\mathcal{N}=1 \mathrm{SYM}$ to its $Z_{2 N}$ subgroup, this discrete global symmetry suffices to forbid a gaugino mass. Gaugino condensation, $\langle\lambda \lambda\rangle \neq 0$, spontaneously breaks $Z_{2 N} \rightarrow Z_{2}$.

However, there have been no GW studies of $\mathcal{N}=1$ SYM for most of the past decade [99101]. Instead, current work uses improved Wilson fermions and fine-tunes the gaugino mass to recover both chiral symmetry and susy in the continuum limit. One major effort by the DESYMünster-Regensburg-Jena Collaboration, currently using clover improvement, has made significant progress in recent years [102-109]. A second group recently began exploring a SYM analogue of the twisted-mass fermion action [110], aiming to improve the formation of composite supermultiplets at non-zero gaugino masses and lattice spacings, and thereby gain better control over the chiral and continuum extrapolations.

The larger number of dimensions requires considering much smaller $N \ll 10$ compared to the lower-dimensional work discussed above, to keep computational costs under control. Current efforts study only gauge groups SU(2) [102 - 106] and SU(3) [107-111]. Figure 4 of Ref. [107] shows recent SU(3) results from Ref. [107] for the masses of two composite states expected to form (part of) a degenerate multiplet in the supersymmetric continuum chiral limit $[112,113]$ : the $0^{++}$'glueball' and the fermionic 'gluino-glue' particle. Even at a fixed lattice spacing the chiral extrapolations of these masses agree within uncertainties. These signs of supermultiplet formation appear much clearer compared to earlier SU(2) results [105], presumably due to either or both the larger $N$ and the use of clover improvement instead of stout smearing.

The chiral extrapolations in Fig. 4 of Ref. [107] are carried out by taking the $m_{\pi}^{2} \rightarrow 0$ limit for an 'adjoint pion' defined in partially quenched chiral perturbation theory [114]. While two-point functions for the physical composite states of $\mathcal{N}=1 \mathrm{SYM}$ all involve fermion-line-disconnected diagrams, $m_{\pi}$ is measured from just the connected part of the correlator for the $\eta^{\prime}$-like 'gluinoball'. Supersymmetric Ward identities provide an alternative means to determine the critical $\kappa_{c}$ corresponding to the chiral limit. The difference between these two determinations of $\kappa_{c}$ can be considered a measure of the susy-breaking discretization artifacts, which is shown for two lattice spacings in Fig. 2 of Ref. [108]. The two available points are consistent with the artifacts vanishing $\propto a^{2}$ as expected for clover fermions, supporting the restoration of susy in the chiral continuum limit.

Many other lattice $\mathcal{N}=1 \mathrm{SYM}$ investigations may be carried out. These include explorations of the finite-temperature phase diagram, with Refs. $[102,115]$ reporting that deconfinement (spontaneous center symmetry breaking) and chiral symmetry restoration appear to occur at the same temperature, which was not known a priori. Refs. [103, 109] investigate the phase diagram on 
$\mathbb{R}^{3} \times S^{1}$ with a small radius for the compactified temporal direction. Comparing thermal and periodic boundary conditions (BCs) for the gauginos, they find evidence that periodic BCs allow the confined, chirally broken phase to persist for weak couplings where analytic semi-classical methods [116] may be reliable. In addition, there is ongoing work to construct and apply a SYM gradient flow [117-119]. Finally, given the progress in algorithms and computing hardware over the past decade, it seems worthwhile to revisit calculations with GW fermions, which could complement and check the ongoing Wilson-fermion work.

\section{Maximally supersymmetric Yang-Mills $(\mathcal{N}=4 \mathbf{S Y M})$ in four dimensions}

In Sec. 1 we discussed why the twisted (and orbifolded) constructions of SYM with exact susy at non-zero lattice spacing require $Q \geq 2^{d}$ supercharges. In $d=4$ dimensions, this constraint picks out another special case, $\mathcal{N}=4 \mathrm{SYM}$ with $Q=16$, for which a single 'twisted-scalar' supercharge $\mathcal{Q}$ is preserved. This theory consists of a $\mathrm{SU}(N)$ gauge field, four Majorana fermions and six real scalars, all massless and transforming in the adjoint rep of $\mathrm{SU}(N)$ as usual. Thanks to its many supersymmetries, large $\mathrm{SU}(4)_{R}$ symmetry and conformal symmetry, $\mathcal{N}=4 \mathrm{SYM}$ is widely studied throughout theoretical physics. It is the conformal field theory of the original AdS/CFT holographic duality [120], and provided early insight into S-duality [121]. Lattice field theory in principle enables non-perturbative investigations of this theory even away from the large$N$ planar regime.

On the lattice, the bosonic fields are combined into five-component complexified gauge links, requiring the $A_{4}^{*}$ lattice with five basis vectors symmetrically spanning $4 \mathrm{~d}$ [2, 57-60]. A single fine-tuning of a marginal operator may be required to recover the continuum twisted rotation symmetry from the $S_{5}$ point-group symmetry of the $A_{4}^{*}$ lattice, which in turn restores the 15 supersymmetries broken by the lattice discretization [15, 122, 123]. Most numerical calculations so far fix the corresponding coefficient to its classical value. These calculations also have to regulate flat directions in both the $\mathrm{SU}(N)$ and $\mathrm{U}(1)$ sectors. A simple (soft $\mathcal{Q}$-breaking) scalar potential suffices to lift the $\mathrm{SU}(N)$ flat directions, and is removed in continuum extrapolations. The $\mathrm{U}(1)$ sector is more challenging, and ongoing work is searching for good ways to handle it [63-65]. The results shown in Fig. 1 lift the U(1) flat directions by modifying the moduli equations in a way that preserves the $\mathcal{Q}$ susy. At least for 't Hooft couplings $\lambda_{\text {lat }} \leq 2$ this results in effective $\mathcal{O}(a)$ improvement indicated by $\mathcal{Q}$ Ward identity violations vanishing $\propto a^{2}$ in the continuum limit [63]. The resulting lattice action is rather complicated, motivating the public development of high-performance parallel code [62] at github. com/daschaich/susy.

Figure 1 presents preliminary results from ongoing lattice $\mathcal{N}=4 \mathrm{SYM}$ calculations. The top plot considers the static potential $V(r)$, which is found to be coulombic at all accessible 't Hooft couplings [61, 124, 125], as expected. Fitting (tree-level-improved [125]) lattice data to the Coulomb potential $V(r)=A-C / r$ predicts the Coulomb coefficient $C(\lambda)$ shown in the figure. There is a famous holographic prediction [126, 127] that in the regime $N \rightarrow \infty$ and $\lambda \rightarrow \infty$ with $\lambda \ll N$ this quantity should behave as $C(\lambda) \propto \sqrt{\lambda}$ up to $\mathcal{O}\left(\frac{1}{\sqrt{\lambda}}\right)$ corrections, and more general analytic results have been obtained in the $N=\infty$ planar limit [128]. The lattice results for $N \leq 4$ and $\lambda_{\text {lat }} \leq 2$ do not show such behavior and instead look consistent with leading-order perturbation theory. The dashed black line is a fit of the $\mathrm{U}(4)$ data to the leading perturbative expression $C(\lambda)=b \lambda_{\text {lat }} /(4 \pi)$, where the fit parameter $b=0.795(13)$ converts the input lattice 
't Hooft coupling to the expected continuum normalization. Higher-order perturbative corrections for $C(\lambda)$ are suppressed by powers of $\frac{\lambda}{2 \pi^{2}}$ [129-131], suggesting that this apparent leading-order behavior for $\lambda_{\text {lat }} \leq 2$ should not be surprising.

The bottom plot of Fig. 1 considers the scaling dimension $\Delta_{K}(\lambda)=2+\gamma_{K}(\lambda)$ of the Konishi operator $\mathcal{O}_{K}=\sum_{I} \operatorname{Tr}\left[X^{I} X^{I}\right]$, where $X^{I}$ are the scalar fields (obtained from a polar decomposition of the complexified lattice gauge links). There are perturbative [132-134] and holo-
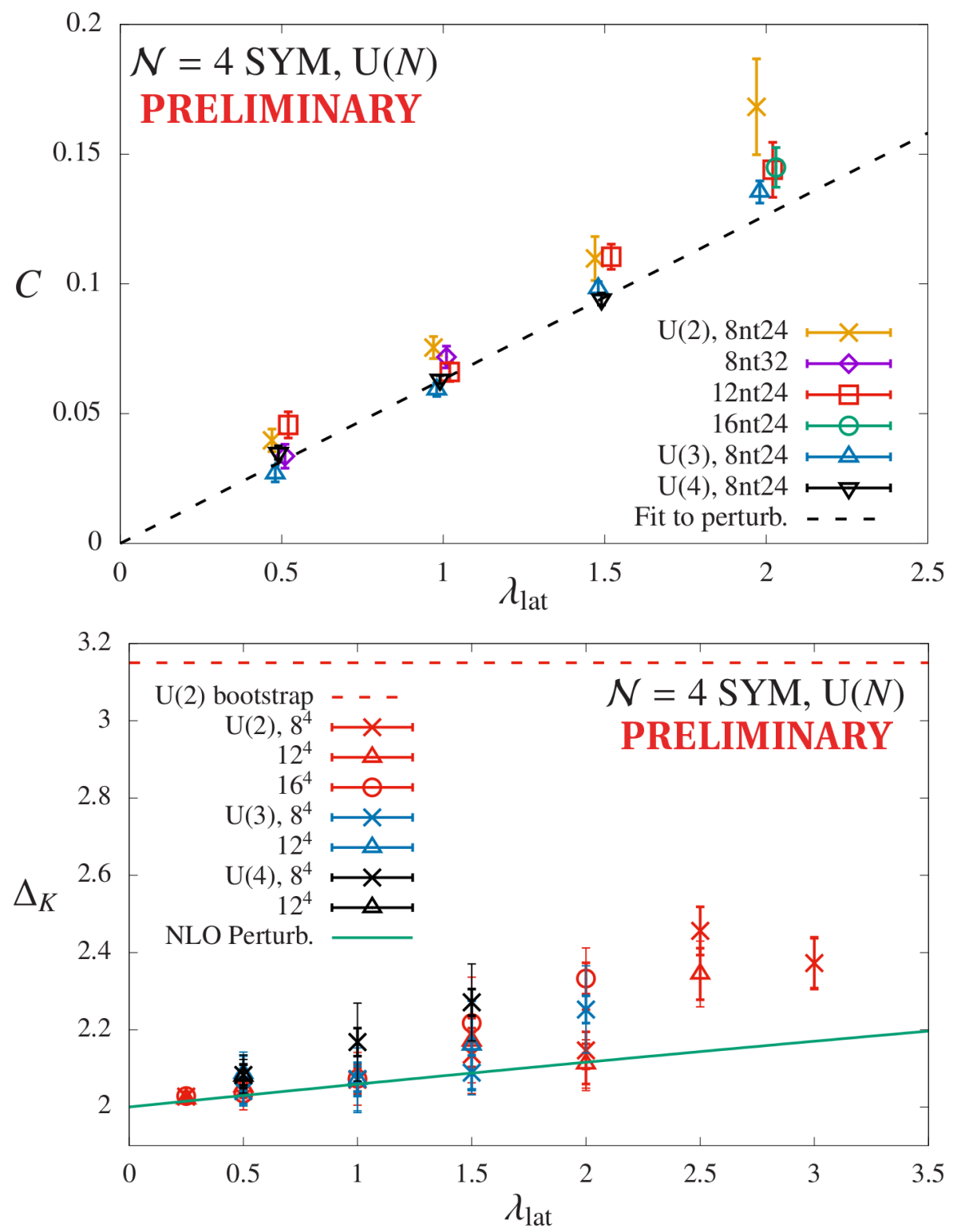

Figure 1: Preliminary results from ongoing $4 \mathrm{~d}$ lattice $\mathcal{N}=4 \mathrm{SYM}$ calculations with gauge groups $\mathrm{U}(2)$, $\mathrm{U}(3)$ and U(4). Top: The static potential Coulomb coefficient, from $L^{3} \times N_{t}$ lattices with $L \leq 16$ and $N_{t} \leq 32$, appears consistent with leading-order perturbation theory (black dashed line) for $\lambda_{\text {lat }} \leq 2$. Bottom: The Konishi scaling dimension, from MCRG stability matrix analyses of $L^{4}$ lattices with $L \leq 16$, also appears consistent with perturbation theory (and well below bootstrap bounds) for $\lambda_{\text {lat }} \lesssim 3$. 
graphic $[135,136]$ predictions for $\Delta_{K}$. The former are also relevant for the strong-coupling regime $\lambda \gg N$ [137], due to the conjectured S-duality of the theory under $\frac{4 \pi N}{\lambda} \longleftrightarrow \frac{\lambda}{4 \pi N}$. In addition, the superconformal bootstrap program has obtained bounds on the maximum value $\gamma_{K}$ can reach across all $\lambda[138,139]$. The lattice results in this figure for $\lambda_{\text {lat }} \lesssim 3$ again appear consistent with perturbation theory. They are obtained from Monte Carlo renormalization group (MCRG) stability matrix analyses [140], with systematic uncertainties estimated by varying the number of interpolating operators in the stability matrix (with different operators obtained by using different amounts of smearing). Additional systematic uncertainties still to be quantified include sensitivity to the lattice volume and the number of RG blocking steps. The stability matrix also includes the related 'SUGRA' or $20^{\prime}$ operator $\mathcal{O}_{S}^{I J}=\operatorname{Tr}\left[X^{\{I} X^{J\}}\right]$, whose scaling dimension $\Delta_{S}=2$ is protected.

Current work only scratches the surface of the investigations that could in principle be pursued by lattice $\mathcal{N}=4 \mathrm{SYM}$. One important task is to push existing studies to stronger 't Hooft couplings, in order to make contact with holographic predictions and ideally investigate the behavior of the system around the S-dual point $\lambda_{\text {sd }}=4 \pi N$. The discussion of sign problems in the next section suggests that this is likely to be challenging. Alternately, S-duality may be studied at weak coupling by adjusting the scalar potential to move the system onto the Coulomb branch of the moduli space where its $\mathrm{U}(N)$ gauge invariance is higgsed to $\mathrm{U}(1)^{N}$. S-duality then relates the masses of the $\mathrm{U}(1)$-charged elementary ' $W$ bosons' and the magnetically charged topological 't Hooft-Polyakov monopoles [121], each of which may be accessible from lattice calculations [141]. The finitetemperature behavior of lattice $\mathcal{N}=4 \mathrm{SYM}$ will also be interesting to explore. In particular, there is motivation [7] to study the free energy, for which the weak-coupling perturbative prediction [142] and the holographic strong-coupling calculation [135] differ by a factor of $\frac{3}{4}$.

\section{Challenges for the future}

Although the recent progress of lattice susy is substantial, it is largely concentrated in the three areas discussed above where significant simplifications are possible. Within those three areas we have already considered several compelling directions for future work, ranging from improved control over large- $N$ continuum extrapolations in lower dimensions, to revisiting $\mathcal{N}=1 \mathrm{SYM}$ with GW fermions, and reaching stronger 't Hooft couplings in $\mathcal{N}=4$ SYM calculations. In addition, it will be important for efforts to expand beyond these domains and tackle more challenging subjects where such simplifications do not appear to be available, such as SQCD.

Supersymmetric QCD: Adding matter multiplets ('quarks' and 'squarks' not necessarily in the fundamental rep) to the $4 \mathrm{~d}$ lattice $\mathcal{N}=1 \mathrm{SYM}$ work discussed in Sec. 2 would enable investigations of many important phenomena, including (metastable) dynamical susy breaking, conjectured electric-magnetic dualities and RG flows to known conformal IR fixed points. The downside is that many more susy-violating operators appear, and the fine-tuning challenge becomes enormously harder. Even exploiting the continuum-like flavor symmetries offered by GW fermions, Ref. [3] counts $\mathcal{O}(10)$ operators to be fine-tuned, depending on the gauge group and matter content. In this context working with GW fermions appears to be especially strongly motivated. Refs. [3, 14] argue that this may allow most or all of the scalar masses, Yukawas and quartic couplings to be fine-tuned "offline" through multicanonical reweighting, which could vastly reduce computational costs. 
That said, as in the case of $\mathcal{N}=1 \mathrm{SYM}$, work currently underway uses Wilson fermions and has to face the full fine-tuning head-on. One tactic for approaching this challenge is to use lattice perturbation theory to guide numerical calculations [143, 144]. Another is to omit the scalar fields at first, and warm up by studying the gauge-fermion theory including both (adjoint) gauginos and (fundamental) quarks [145], which also provides connections to composite Higgs investigations that are reviewed by another contribution to these proceedings [146].

Following the logic of Sec. 1, it may prove advantageous to first investigate simpler systems in fewer than four dimensions. In 0+1 dimensions, for example, Refs. [147-149] consider the Berkooz-Douglas matrix model [150], which adds $N_{f}$ fundamental multiplets to $Q=16$ SYM QM (preserving half of the supercharges in the continuum). As for the case of $2 \mathrm{~d}$ and $3 \mathrm{~d}$ SYM, more effort has been devoted to constructing clever lattice formulations of $2 \mathrm{~d}$ and $3 \mathrm{~d}$ SQCD [151-156] compared to carrying out numerical calculations [157]. That one numerical calculation [157] uses a quiver construction of $2 \mathrm{~d} Q=4$ SQCD that still preserves one of the supercharges at non-zero lattice spacing [151, 152]. The starting point is $3 \mathrm{~d} 8$-supercharge SYM on a lattice with only two slices in the third direction. The twisted formulation can be generalized to have different gauge groups $\mathrm{U}(N)$ and $\mathrm{U}(F)$ on each slice, with the bosonic and fermionic fields that connect the two slices transforming in the bifundamental rep of $\mathrm{U}(N) \times \mathrm{U}(F)$. Decoupling the $\mathrm{U}(F)$ slice then leaves behind a $2 \mathrm{~d} \mathrm{U}(N)$ theory with half the supercharges $(Q=4)$ and $F$ massless fundamental matter multiplets. This same procedure works for $Q=8 \mathrm{SQCD}$ in two and three dimensions [155], and may be generalizable to higher reps [156]. Ref. [157] compares U(2) SQCD with $F=3$ vs. U(3) SQCD with $F=2$, observing dynamical susy breaking for $N>F$ and checking that the goldstino is consistent with masslessness in infinite volume.

Sign problems: Another challenge is that some of the systems discussed above may suffer from a sign problem, at least in certain regimes. Integrating over the gauginos produces the pfaffian of the fermion operator, which can fluctuate in sign even when the determinant would be positive. Writing a generic complex pfaffian as $\operatorname{pf} \mathcal{D}=|\operatorname{pf} \mathcal{D}| e^{i \alpha}$, only its magnitude is included in the 'phase-quenched' RHMC studies presented above. The phase-quenched observables $\langle\mathcal{O}\rangle_{\mathrm{pq}}$ need to be reweighted, $\langle\mathcal{O}\rangle=\langle\mathcal{O}\rangle_{\mathrm{pq}} /\left\langle e^{i \alpha}\right\rangle_{\mathrm{pq}}$, with a sign problem appearing when $\left\langle e^{i \alpha}\right\rangle_{\mathrm{pq}}=Z / Z_{\mathrm{pq}}$ vanishes within statistical uncertainties [158]. In lattice calculations with periodic BCs for all fields, the partition function $Z$ is the Witten index and must vanish for any theory that can exhibit SSB [159], implying a severe sign problem.

For Wilson-fermion $\mathcal{N}=1 \mathrm{SYM}$ the pfaffian is real and its sign can be computed efficiently [160]. Recent clover calculations report $\left\langle e^{i \alpha}\right\rangle_{\mathrm{pq}} \approx 1$, with the situation improving further as the lattice spacing decreases [107]. However, $\left\langle e^{i \alpha}\right\rangle_{\mathrm{pq}}$ is expected to decrease exponentially in the lattice volume, and the situation is likely to be worse for SQCD. Directly evaluating the pfaffian is much more computationally expensive, and has been done mostly for SYM QM and $d=2$ SYM, where sign problems also appear to be well under control [35, 37, 79, 80, 85, 94, 96].

Figure 2 presents results for the pfaffian phase of lattice $\mathcal{N}=4$ SYM in four dimensions, adapted from Refs. [123, 161], where only small $N$ and small lattice volumes are computationally accessible. In the top plot, only small per-mille-level phase fluctuations are observed on all accessible volumes with fixed 't Hooft coupling $\lambda_{\text {lat }}=0.5$. In particular, the expected exponential suppression of $\left\langle e^{i \alpha}\right\rangle_{\mathrm{pq}}$ with the lattice volume is not visible; instead the largest volumes for gauge 
group $\mathrm{U}(2)$ produce results that are constant within uncertainties. In the bottom plot, however, we see phase fluctuations increasing significantly for stronger 't Hooft couplings $\lambda_{\text {lat }} \gtrsim 2$. This appears to be one of the main obstacles to reaching the stronger couplings of interest in order to directly probe holography and S-duality, with calculations using this lattice action largely limited to $\lambda_{\text {lat }} \lesssim 4$.

Final remarks: Non-perturbative lattice investigations of supersymmetric QFTs are important and challenging, making this a field in which we can expect to see a great deal more work in the
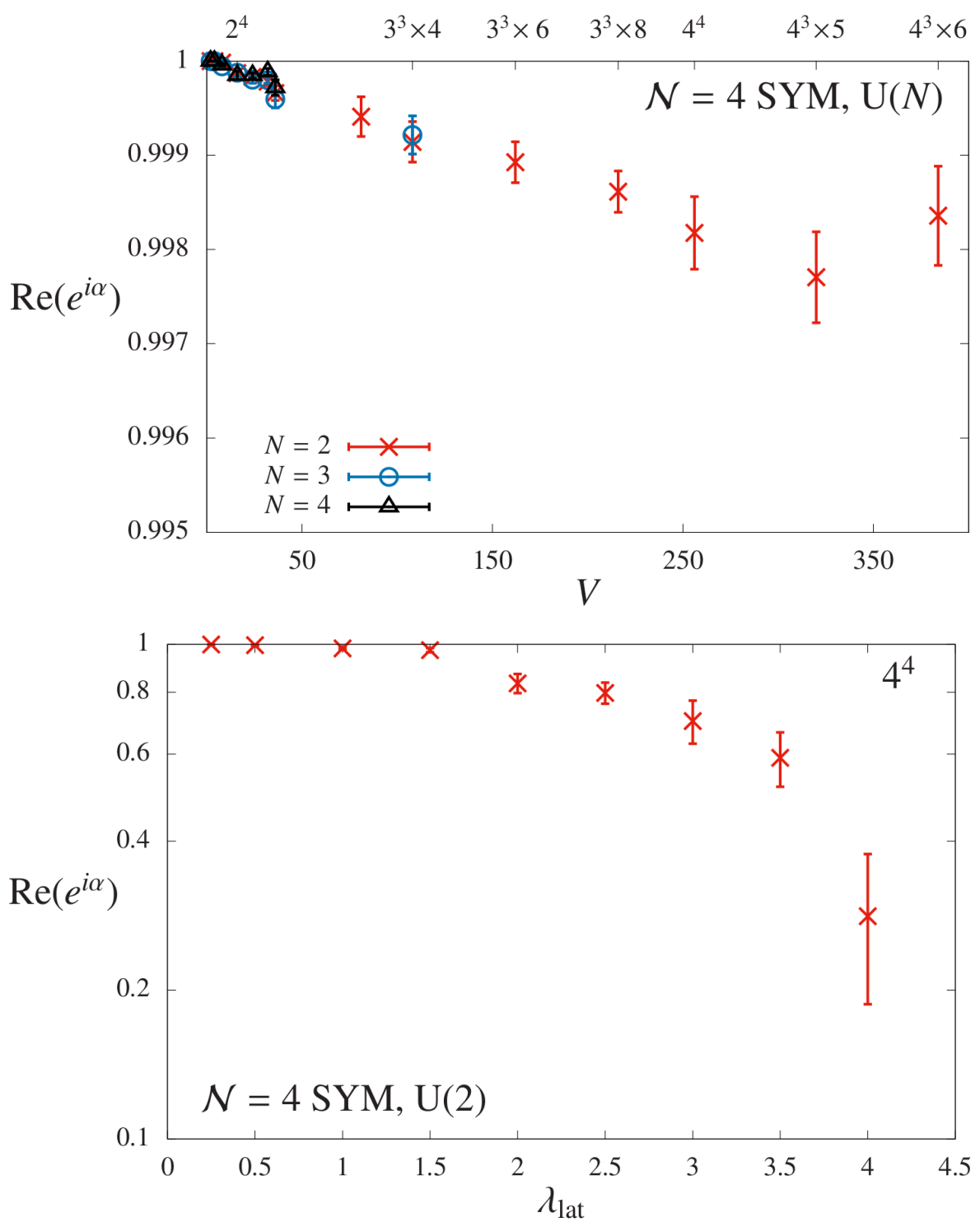

Figure 2: Results for the phase of the pfaffian $\left\langle\operatorname{Re}\left(e^{i \alpha}\right)\right\rangle_{\mathrm{pq}} \approx\left\langle e^{i \alpha}\right\rangle_{\mathrm{pq}}$ from lattice $\mathcal{N}=4 \mathrm{SYM}$ in four dimensions. Top: With fixed 't Hooft coupling $\lambda_{\text {lat }}=0.5$, only per-mille-level fluctuations are observed for $\mathrm{U}(N)$ gauge groups with $N=2,3$ and 4, up to the largest accessible volumes. Adapted from Ref. [123]. Bottom: On a fixed $4^{4}$ lattice volume, the phase fluctuations increase significantly for stronger couplings $\lambda_{\text {lat }} \gtrsim 2$, obstructing studies of $\lambda_{\text {lat }} \gtrsim 4$ with this lattice action. Adapted from Ref. [161]. 
future. It is encouraging that there has been so much recent progress in lattice studies of $4 \mathrm{~d} \mathcal{N}=1$ SYM and $\mathcal{N}=4 \mathrm{SYM}$, along with their dimensional reductions to $d<4$. This brief overview has also omitted coverage of advances in other areas, including theories without gauge invariance [162-167], the lattice regularization of the Green-Schwarz superstring worldsheet sigma model [168 - 170], and proposed lattice formulations of a mass-deformed $4 \mathrm{~d} \mathcal{N}=2^{*} \mathrm{SYM}$ theory with $Q=8$ [171] and of 5d $Q=16$ SYM [172]. While there are clear challenges that will be difficult to overcome, in particular concerning supersymmetric QCD and sign problems, overall the prospects of lattice susy are bright, with many compelling directions for future investigations.

ACKNOWLEDGMENTS: I thank the organizers of Lattice 2018 for the invitation to present this overview, and for all their work. E. Rinaldi, J. Nishimura, H. So, M. Costa, G. Bergner and B. Wellegehausen provided helpful information. I have benefited from collaboration on lattice susy with S. Catterall, P. Damgaard, T. DeGrand, J. Giedt, R. Jha, A. Joseph and T. Wiseman.

\section{References}

[1] P. H. Dondi and H. Nicolai, Nuovo Cim. A41 (1977) 1.

[2] S. Catterall, D. B. Kaplan and M. Unsal, Phys. Rept. 484 (2009) 71-130 [0 903.4881$].$

[3] J. Giedt, IJMPA 24 (2009) 4045-4095 [0903.2443].

[4] A. Joseph, IJMPA 30 (2015) 1530054 [1509.01440].

[5] D. Kadoh, PoS LATTICE2015 (2016) 017 [1607. 01170].

[6] G. Bergner and S. Catterall, IJMPA 31 (2016) 1643005 [1603.04478].

[7] M. Hanada, IJMPA 31 (2016) 1643006 [1604.05421].

[8] M. Kato, M. Sakamoto and H. So, JHEP 0805 (2008) 057 [0 803 . 3121].

[9] G. Bergner, JHEP 1001 (2010) 024 [0 909.4791$].$

[10] M. Kato, M. Sakamoto and H. So, PTEP 2017 (2017) 043B09 [1609. 08793].

[11] M. Kato, M. Sakamoto and H. So, JHEP 1305 (2013) 089 [1303 . 4472 ].

[12] A. D'Adda, N. Kawamoto and J. Saito, JHEP 1712 (2017) 089 [1 706.02615 ].

[13] M. Kato, M. Sakamoto and H. So, 1809.02379.

[14] J. W. Elliott, J. Giedt and G. D. Moore, PRD 78 (2008) 081701 [0 806.0013 ].

[15] S. Catterall and J. Giedt, JHEP 1411 (2014) 050 [1408 . 7067].

[16] J. Giedt, R. Koniuk, E. Poppitz and T. Yavin, JHEP 0412 (2004) 033 [hep-lat / 0410041 ].

[17] G. Bergner, T. Kaestner, S. Uhlmann and A. Wipf, Annals Phys. 323 (2008) 946 [0 705 . 2212].

[18] J. Giedt, A. Lipstein and P. Martin, PoS LATTICE2018 (2018, to appear) [1811.00516].

[19] M. Hanada, J. Nishimura and S. Takeuchi, PRL 99 (2007) 161602 [0 706.1647 ].

[20] S. Catterall and T. Wiseman, JHEP 0712 (2007) 104 [0 0706 . 3518].

[21] K. N. Anagnostopoulos, M. Hanada, J. Nishimura et al., PRL 100 (2008) 021601 [0 707 . 4454 ].

[22] M. Hanada, A. Miwa, J. Nishimura and S. Takeuchi, PRL 102 (2009) 181602 [0811.2081].

[23] M. Hanada, Y. Hyakutake, J. Nishimura and S. Takeuchi, PRL 102 (2009) 191602 [0811. 3102].

[24] M. Hanada, J. Nishimura, Y. Sekino and T. Yoneya, PRL 104 (2010) 151601 [0 911.1623 ].

[25] M. Hanada, J. Nishimura, Y. Sekino and T. Yoneya, JHEP 1112 (2011) 020 [1108 . 5153].

[26] M. Hanada, Y. Hyakutake, G. Ishiki and J. Nishimura, Science 344 (2014) 882-885 [1311 . 560 7].

[27] J. Maldacena and A. Milekhin, JHEP 1804 (2018) 084 [1802 . 00428].

[28] E. Berkowitz, M. Hanada, E. Rinaldi and P. Vranas, JHEP 1806 (2018) 124 [18 02.02985$].$

[29] E. Berkowitz, E. Rinaldi, M. Hanada et al., 1606.04948 ; PRD 94 (2016) 094501 [1606. 04951 ].

[30] B. de Wit, J. Hoppe and H. Nicolai, NPB 305 (1988) 545.

[31] T. Banks, W. Fischler, S. H. Shenker and L. Susskind, PRD 55 (1997) 5112 [hep-th/ 9610043 ].

[32] W. Taylor, NATO Sci. Ser. C 556 (2000) 91-178 [hep-th / 0002016 ]. 
[33] B. Ydri, Matrix Models of String Theory, IOP Publishing, 2018 [arXiv: 1708.00734 ].

[34] S. Catterall and T. Wiseman, PRD 78 (2008) 041502 [0803.4273].

[35] S. Catterall and T. Wiseman, JHEP 1004 (2010) 077 [0 909.4947 ].

[36] D. Kadoh and S. Kamata, 1503.08499.

[37] V. G. Filev and D. O'Connor, JHEP 1605 (2016) 167 [1506.01366].

[38] M. Hanada, Y. Hyakutake, G. Ishiki and J. Nishimura, PRD 94 (2016) 086010 [1603.00538].

[39] M. Hanada and I. Kanamori, PRD 80 (2009) 065014 [0 907 . 4966].

[40] K. Steinhauer and U. Wenger, JHEP 1412 (2014) 044 [1410 . 0235].

[41] Z. Ambroziński and P. Korcyl, PoS LATTICE2014 (2015) 253 [1411. 6904 ].

[42] G. Bergner, H. Liu and U. Wenger, PoS LATTICE2016 (2016) 395 [1612 . 04291 ].

[43] E. Rinaldi, E. Berkowitz, M. Hanada, J. Maltz, P. Vranas, JHEP 1802 (2018) 042 [1709 . 01932].

[44] P. V. Buividovich, M. Hanada and A. Schäfer, 1810.03378.

[45] D. E. Berenstein, J. M. Maldacena and H. S. Nastase, JHEP 0204 (2002) 013 [hep-th/ 0202021 ].

[46] S. Catterall and G. van Anders, JHEP 1009 (2010) 088 [1003.4952].

[47] M. Honda, G. Ishiki, S.-W. Kim, J. Nishimura, A. Tsuchiya, JHEP 1311 (2013) 200 [1308 . 3525].

[48] Y. Asano, V. G. Filev, S. Kováčik and D. O'Connor, JHEP 1807 (2018) 152 [18 05.05314 ].

[49] M. S. Costa, L. Greenspan, J. Penedones and J. Santos, JHEP 1503 (2015) 069 [1411. 5541 ].

[50] S. Catterall and A. Joseph, CPC 183 (2012) 1336-1353 [1108 . 1503].

[51] J. Giedt and A. E. Lipstein, JHEP 1803 (2018) 162 [1711. 05203].

[52] F. Sugino, JHEP 0401 (2004) 015 [hep-lat/0311021].

[53] F. Sugino, JHEP 0403 (2004) 067 [hep-lat/ 0401017$].$

[54] S. Catterall, JHEP 0411 (2004) 006 [hep-lat/ 0410052$].$

[55] A. G. Cohen, D. B. Kaplan, E. Katz and M. Ünsal, JHEP 0308 (2003) 024 [hep-lat/ 0302017 ].

[56] A. G. Cohen, D. B. Kaplan, E. Katz and M. Ünsal, JHEP 0312 (2003) 031 [hep-lat/ 0307012 ].

[57] D. B. Kaplan and M. Ünsal, JHEP 0509 (2005) 042 [hep-lat / 0503039 ].

[58] M. Ünsal, JHEP 0610 (2006) 089 [hep-th / 0603046 ].

[59] S. Catterall, JHEP 0801 (2008) 048 [0 712.2532$].$

[60] P. H. Damgaard and S. Matsuura, PLB 661 (2008) 52-56 [0801.2936].

[61] S. Catterall, D. Schaich, P. Damgaard, T. DeGrand, J. Giedt, PRD 90 (2014) 065013 [1 405.0644 ].

[62] D. Schaich and T. DeGrand, CPC 190 (2015) 200-212 [1410.6971].

[63] S. Catterall and D. Schaich, JHEP 1507 (2015) 057 [1505. 03135].

[64] S. Catterall, R. G. Jha, D. Schaich and T. Wiseman, PRD 97 (2018) 086020 [170 9.07025$].$

[65] S. Catterall, J. Giedt and R. G. Jha, 1808.04735.

[66] D. Kadoh and H. Suzuki, PLB 682 (2010) 466-471 [0 908.2274$].$

[67] M. Hanada, S. Matsuura and F. Sugino, PTP 126 (2011) 597-611 [1 004 . 5513].

[68] M. Hanada, S. Matsuura and F. Sugino, NPB 857 (2012) 335-361 [1109.6807].

[69] S. Matsuura, T. Misumi and K. Ohta, PTEP 2014 (2014) 123B01 [1408.6998].

[70] M. Hanada, D. Kadoh, S. Matsuura and F. Sugino NPB 929 (2018) 266-297 [1711. 02319].

[71] S. Matsuura and F. Sugino JHEP 1404 (2014) 088 [1402.0952].

[72] F. Sugino JHEP 0501 (2005) 016 [hep-lat/ 0410035 ].

[73] H. Suzuki JHEP 0709 (2007) 052 [0 706 . 1392].

[74] I. Kanamori, H. Suzuki and F. Sugino, PRD 77 (2008) 091502 [0711.2099].

[75] I. Kanamori, F. Sugino and H. Suzuki, PTP 119 (2008) 797-827, [0 711.2132$].$

[76] I. Kanamori and H. Suzuki, NPB 811 (2009) 420-437 [0809 . 2856].

[77] I. Kanamori and H. Suzuki, PLB 672 (2009) 307-311 [0811.2851].

[78] I. Kanamori, PRD 79 (2009) 115015 [0902.2876].

[79] M. Hanada and I. Kanamori, JHEP 1101 (2011) 058 [1010.2948].

[80] S. Kamata, S. Matsuura, T. Misumi and K. Ohta, PTEP 2016 (2016) 123B01 [1607. 01260]. 
[81] E. Giguère and D. Kadoh, JHEP 1505 (2015) 082 [1503.04416].

[82] D. Kadoh, PoS LATTICE2016 (2017) 033 [1702.01615].

[83] S. Catterall, JHEP 0901 (2009) 040 [0811.1203].

[84] S. Catterall, A. Joseph and T. Wiseman, JHEP 1012 (2010) 022 [1008.4964].

[85] S. Catterall, R. Galvez, A. Joseph and D. Mehta, JHEP 1201 (2012) 108 [1112 . 3588].

[86] R. G. Jha, PoS LATTICE2018 (2018, to appear) [1809.00797].

[87] O. Aharony, J. Marsano, S. Minwalla and T. Wiseman, CQG 21 (2004) 5169 [hep-th/ 0406210 ].

[88] N. Kawahara, J. Nishimura and S. Takeuchi, JHEP 0710 (2007) 097 [0 706 . 3517].

[89] G. Mandal, M. Mahato and T. Morita, JHEP 1002 (2010) 034 [0910 . 4526].

[90] T. Azuma, T. Morita and S. Takeuchi, PRL 113 (2014) 0916031403.7764$].$

[91] Ó. J. C. Dias, J. E. Santos and B. Way, JHEP 1706 (2017) 029 [1702 . 07718].

[92] E. Witten, $N P B 460$ (1996) 335-350 [hep-th/9510135].

[93] H. Fukaya, I. Kanamori, H. Suzuki et al., PTP 116 (2007) 1117 [hep-th/ 0609049 ].

[94] D. August, B. H. Wellegehausen and A. Wipf, 1802.07797.

[95] K. Hori and D. Tong, JHEP 0705 (2007) 079 [hep-th/ 0609032 ].

[96] S. Catterall, R. G. Jha and A. Joseph, PRD 97 (2018) 054504 [1801. 00012 ].

[97] G. Curci and G. Veneziano, NPB 292 (1987) 555-572.

[98] H. Suzuki, NPB 861 (2012) 290-320 [1202 .2598].

[99] J. Giedt, R. Brower, S. Catterall, G. Fleming, P. Vranas, PRD 79 (2009) 025015 [0 810 . 574 6]. [100] M. G. Endres, PRD 79 (2009) 094503 [0 902 . 4267].

[101] JLQCD Collaboration: S. W. Kim, H. Fukaya et al., PoS LATTICE2011 (2011) 069 [1111.2180]. [102] G. Bergner, P. Giudice, G. Münster, S. Piemonte et al., JHEP 1411 (2014) 049 [1405 . 3180].

[103] G. Bergner and S. Piemonte, JHEP 1412 (2014) 133 [1410 . 3668].

[104] G. Bergner, P. Giudice, I. Montvay, G. Münster et al., EPJ Plus 130 (2015) 229 [1411. 6995]. [105] G. Bergner, P. Giudice, G. Münster, I. Montvay et al., JHEP 1603 (2016) 080 [1512 . 0 7014].

[106] S. Ali, G. Bergner, H. Gerber, P. Giudice et al., EPJ Web Conf. 175 (2018) 08016 [1710 . 07464 ].

[107] S. Ali, G. Bergner, H. Gerber, P. Giudice, I. Montvay et al., JHEP 1803 (2018) 113 [1801. 08062 ].

[108] S. Ali, H. Gerber, I. Montvay, G. Münster, S. Piemonte et al., EPJC 78 (2018) 404 [1802 . 0 7067 ].

[109] G. Bergner, S. Piemonte and M. Ünsal, 1806.10894.

[110] M. Steinhauser, A. Sternbeck, B. Wellegehausen and A. Wipf, EPJ Web Conf. 175 (2018) 08022 [1711. 0 5086]; PoS LATTICE2018 (2018) 211 [arXiv:1811.01785].

[111] S. Ali, G. Bergner, H. Gerber, C. López et al., PoS LATTICE2018 (2018) 207 [1811 . 02297].

[112] G. Veneziano and S. Yankielowicz, PLB 113 (1982) 231.

[113] G. R. Farrar, G. Gabadadze and M. Schwetz, PRD 58 (1998) 015009 [hep-th/9711166].

[114] G. Münster and H. Stüwe, JHEP 1405 (2014) 034 [1 402 . 6616].

[115] G. Bergner, C. López and S. Piemonte, PoS LATTICE2018 (2018) 212 [1811. 02270].

[116] E. Poppitz, T. Schäfer and M. Ünsal, JHEP 1210 (2012) 115 [1205. 0290].

[117] D. Kadoh and N. Ukita, PoS LATTICE2018 (2018, to appear); 1812.02351.

[118] K. Hieda, A. Kasai, H. Makino and H. Suzuki, PTEP 2017 (2017) 063B03 [1 703.04802 ].

[119] A. Kasai, O. Morikawa and H. Suzuki, [1808.07300].

[120] J. M. Maldacena, Adv. Theor. Math. Phys. 2 (1998) 231-252, hep-th/9711200].

[121] H. Osborn, PLB 83 (1979) 321-326.

[122] S. Catterall, J. Giedt and A. Joseph, JHEP 1310 (2013) 166 [1306 . 3891$].$

[123] S. Catterall, J. Giedt, D. Schaich et al., PoS LATTICE2014 (2014) 267 [1411. 0166].

[124] S. Catterall, P. Damgaard, T. Degrand, R. Galvez, D. Mehta, JHEP 1211 (2012) 072 [120 9 . 5285].

[125] D. Schaich, S. Catterall, P. H. Damgaard, J. Giedt, PoS LATTICE2016 (2016) 221 [1611. 06561 ].

[126] S.-J. Rey and J.-T. Yee, EPJC 22 (2001) 379-394 [hep-th/ 9803001 ].

[127] J. M. Maldacena, PRL 80 (1998) 4859-4862 [hep-th/9803002]. 
[128] N. Gromov and F. Levkovich-Maslyuk, JHEP 1612 (2016) 122 [1601. 05679].

[129] A. Pineda, $P R D 77$ (2008) 021701 [0 709.2876$].$

[130] M. Stahlhofen, JHEP 1211 (2012) 155 [1209.2122].

[131] M. Prausa and M. Steinhauser, PRD 88 (2013) 025029 [1306. 5566].

[132] F. Fiamberti, A. Santambrogio, C. Sieg and D. Zanon, NPB 805 (2008) 231-266 [08 06 . 20 95].

[133] Z. Bajnok and R. A. Janik, NPB 807 (2009) 625-650 [0807 . 0399].

[134] V. N. Velizhanin, JETP Lett. 89 (2009) 6-9, 0808 . 3832].

[135] S. S. Gubser, I. R. Klebanov and A. M. Polyakov, PLB 428 (1998) 105-114 [hep-th/ 9802109 ].

[136] N. Gromov, V. Kazakov and P. Vieira, PRL 104 (2010) 211601 [0 906.4240 ].

[137] C. Beem, L. Rastelli, A. Sen and B. C. van Rees, JHEP 1404 (2014) 122 [1306 . 3228].

[138] C. Beem, L. Rastelli and B. C. van Rees, PRL 111 (2013) 071601 [1304 . 1803 ].

[139] C. Beem, L. Rastelli and B. C. van Rees, PRD 96 (2017) 046014 [1612 . 02363].

[140] R. H. Swendsen, PRL 42 (1979) 859-861.

[141] J. Giedt, S. Catterall, P. Damgaard and D. Schaich, PoS LATTICE2016 (2016) 209 [18 04.07792 ].

[142] A. Fotopoulos and T. R. Taylor PRD 59 (1999) 061701 [hep-th/9811224].

[143] M. Costa and H. Panagopoulos, PRD 96 (2017) 034507 [1706 . 0 5222]]; 1812.06770.

[144] B. H. Wellegehausen and A. Wipf, PoS LATTICE2018 (2018) 210 [1811. 01784 ].

[145] G. Bergner and S. Piemonte, PoS LATTICE2018 (2018, to appear) [1811. 01797].

[146] O. Witzel PoS LATTICE2018 (2018, to appear).

[147] V. G. Filev and D. O'Connor, JHEP 1605 (2016) 122 [1512.02536].

[148] Y. Asano, V. G. Filev, S. Kováčik and D. O’Connor, JHEP 1701 (2017) 113 [1605. 05597].

[149] Y. Asano, V. G. Filev, S. Kováčik and D. O’Connor, JHEP 1803 (2018) 055 [1612. 09281 ].

[150] M. Berkooz and M. R. Douglas, PLB 395 (1997) 196-202 [hep-th/ 9610236 ].

[151] S. Matsuura, JHEP 0807 (2008) 127 [0805.4491].

[152] F. Sugino, $N P B 808$ (2009) 292-325 [0807.2683].

[153] Y. Kikukawa and F. Sugino, NPB 819 (2009) 76-115 [0811.0916].

[154] D. Kadoh, F. Sugino and H. Suzuki, NPB 820 (2009) 99-115 [0 903.5398 ].

[155] A. Joseph, JHEP 1309 (2013) 046 [1307. 3281]; JHEP 1401 (2014) 093 [1311. 5111].

[156] A. Joseph, JHEP 1407 (2014) 067 [1403.4390].

[157] S. Catterall and A. Veernala, JHEP 1510 (2015) 013 [1505.00467].

[158] P. de Forcrand, PoS LAT2009 (2009) 010 [1 005 . 0539].

[159] E. Witten, $N P B 202$ (1982) 253.

[160] G. Bergner and J. Wuilloud, CPC 183 (2012) 299-304 [1104 .1363].

[161] D. Schaich and S. Catterall, IJMPA 32 (2017) 1747019 [1508.00884].

[162] C. Wozar and A. Wipf, Annals Phys. 327 (2012) 774-807 [1107.3324].

[163] K. Steinhauer and U. Wenger, PRL 113 (2014) 231601 [1410.6665].

[164] D. Baumgartner and U. Wenger, NPB 894 (2015) 223-253 [1412 . 5393 ]; NPB 897 (2015) 39-76 [1503.05232]; NPB 899 (2015) 375-394 [1505.07397].

[165] S. Aoki, K. Kikuchi and T. Onogi, JHEP 1802 (2018) 128 [170 4.03717$].$

[166] D. Kadoh, Y. Kuramashi, Y. Nakamura, R. Sakai et al., JHEP 1803 (2018) 141 [1801. 04183 ].

[167] D. Kadoh and K. Nakayama, NPB 932 (2018) 278-297 [1803.07960]; 1812.10642.

[168] R. W. McKeown and R. Roiban, 1308.4875.

[169] L. Bianchi, M. S. Bianchi, V. Forini, B. Leder, E. Vescovi, JHEP 1607 (2016) 014 [1605. 01726].

[170] V. Forini, in Space-Time-Matter: Analytic and Geometric Structures, 221, 2018 [1712.10301].

[171] A. Joseph, PRD 97 (2018) 094508 [1710.10172].

[172] A. Joseph, JHEP 1606 (2016) 030 [1604.02707]. 